\title{
Correlation Between Radiographic Grading of Osteoarthritis, Pain Severity and Functional Status in Knee Osteoarthritis Patients
}

\author{
Annisa Dwi Khairina, ${ }^{1}$ Marina A. Moeliono, ${ }^{2}$ Andri Reza Rahmadi ${ }^{3}$ \\ ${ }^{1}$ Faculty of Medicine Universitas Padjadjaran, ${ }^{2}$ Department of Physical Medicine and \\ Rehabilitation Faculty of Medicine Universitas Padjadjaran/Dr. Hasan Sadikin General Hospital \\ Bandung, ${ }^{3}$ Department of Internal Medicine Faculty of Medicine Universitas Padjadjaran/Dr. \\ Hasan Sadikin General Hospital Bandung, Indonesia
}

\begin{abstract}
Background: Osteoarthritis is a joint degenerative disease characterized with structural changes of joint. The structural changes can be assessed with radiological assessment. Major clinical manifestations may include pain, inflammation, and stiffness, resulting in limitations for performing daily activities. The purpose of this study was to determine the correlation between radiographic grading of osteoarthritis, pain severity, and lower extremities functional status in patients with knee osteoarthritis.

Methods: The study involved 29 patients with knee osteoarthritis in Dr. Hasan Sadikin General Hospital, Bandung and Bandung District General Hospital during the period of August to October 2014 using the cross sectional method. This study used NRS (Numeric Rating Scale) for pain severity assessment, KellgrenLawrence criteria for radiographic grading of osteoarthritis, and Modified Lower Extremity Funcional Scale for functional status assessment. The data were analyzed using statistical analysis tools. If the data were normally distributed, the correlation test will be carried out by using the Pearson correlation test and if the data were not normally distributed then the Spearman's Correlation test will be used.

Results: Correlation coefficient between severity of pain and functional status is $r=-0,439$ with $p$ value= $0.17(p<0.05)$. There was no significant correlation between radiographic grading of osteoarthritis and functional status $(\mathrm{p}>0.05)$.

Conclusions: The severity of pain correlates with the functional status of patients with osteoarthritis of the knee, while radiographic grading of osteoarthritis does not correlate with functional status.

Keywords: Kellgren-Lawrence criteria, knee osteoarthritis, modified lower extremity functional scale, radiographic grading

\section{Introduction}

Osteoarthritis is a degenerative joint disease characterized by degeneration of the articular cartilage, hypertrophy of bone at the margin, and changes in synovial membrane. ${ }^{1}$ Osteoartritis is the most common disease that is equal to $50-60 \%$ of the total of rheumatic diseases in Indonesia. ${ }^{2}$ Major clinical manifestations of osteoarthritis are pain, swelling, and stiffness. ${ }^{3}$ The World Health Organization reports that $80 \%$ of patients with osteoarthritis will have limitations in movement, and 25\% cannot perform daily activities of live. ${ }^{4}$

Several studies have been conducted to determine the relationship between clinical symptoms with disability and radiographic results using the Western Ontario and McMaster Universities Osteoarthritis Index

(WOMAC) questionnaire. ${ }^{5}$ Besides WOMAC, there are also other measurement tools to measure functional status, namely the Lower Extremity Functional Scale (LEFS). This study used the Lower Extremity Functional Scale because it was recommended by several other studies as a measurement of functional status in osteoarthritis because it is able to distinguish between pain and physical function. ${ }^{6,7}$

The daily living activities of people in Indonesia are slightly different from the community activities in other cultures, therefore, this study used LEFS that has been modified in accordance with daily activities in Indonesia. The purpose of this study was to analyze the correlation between the radiographic grading of osteoarthritis, severity of pain and functional status by the Modified Lower Extremity Functional Scale on patients with knee osteoarthritis.
\end{abstract}

Correspondence: Annisa Dwi Khairina, Faculty of Medicine, Universitas Padjadjaran, Jalan Raya Bandung-Sumedang Km.21, Jatinangor, Sumedang, Indonesia, Email: annisadwikhairina@gmail.com 


\section{Methods}

The study was conducted at the Polyclinic of Physical Medicine and Rehabilitation and the Polyclinic of Rheumatology of Internal Medicine Dr. Hasan Sadikin General Hospital Bandung and Bandung District General Hospital during the period of August to October 2014. The study was approved by the Health Research Ethics committee of the Faculty of Medicine, Universitas Padjadjaran, the Ethics committee of the General Hospital Dr. Hasan Sadikin, and the Ethics committee of the Bandung District General Hospital.

The cross sectional method was used in this study. The sample size was calculated using the formula of correlation analysis that established the value of $\alpha=0.05$, it is worth $1.96 \mathrm{Z} \alpha, \mathrm{Z} \beta$ value $=0.84$, and the minimum correlation value considered significant is 0.4 , then the calculated sample size obtained was 46. Inclusion criteria for this study were patients with clinical and radiological diagnosis of knee osteoarthritis, age range between 50 to 70 years, cooperative and willing to answer questions in the questionnaire. Exclusion criteria were knee osteoarthritis patients who did not have the required data according to the study and patients with a diagnosis other than osteoarthritis in the lower extremities. Patients who had agreed to be involved as study subjects then recorded their identity (name, age, gender, and diagnosis), radiographic grading of osteoarthritis, severity of pain, and functional status.

The radiographic grading of osteoarthritis assessment used the Kellgren-Lawrence criteria, which was divided into four levels of severity. The assessment of radiographic grading was obtained from the results of the radiological diagnosis which has been performed by a radiologist.

The severity of pain was assessed using the Numeric Rating Scale (NRS). The higher the number on a scale of $0-10$ showed more severe levels of pain felt by the patient. The assessment was categorized as no pain $(0)$, mild pain (1-3), moderate pain (4-6), and severe pain (7-10). Patients were asked about the pain during the day they come to the health facilities.

The lower extremity functional status was assessed using the Modified Lower Extremity Functional Scale questionnaire, which was a translation and modification of the Lower Extremity Functional Scale (LEFS). The modification was adjusted to the conditions of people's daily activities in Indonesia and had been validated. The Modified Lower Extremity Functional Scale contained 22 questions about patients' difficulties in performing daily activities. The level of difficulty in performing the activities in the questions were assessed in a scale of $0-4$. The lower the value, the more severe difficulties patients experienced. The value of each question was then summed up and the maximum value of the Modified Lower Extremity Functional Scale obtained was 88 which showed no limitations to perform the activity.

The data were analyzed using statistical analysis tools. If the data were normally distributed, the correlation test will be carried out by using the Pearson correlation test and if the data were not normally distributed then Spearman's Correlation will be used.

\section{Results}

The total respondents during the study period were only 29 patients compared to the need of minimum sample (46 samples). Most of the respondents were female. Regarding age, the average age was 58 yearswith the youngest age was 50 years and the oldest was 70 years.

Based on the Kellgren-Lawrence criteria, there were no subjects with radiographic grade IV and most of the subjects had radiographic grade II (79.3\%). Most of the patients experienced pain in the moderate pain category $(62.1 \%)$.

Results of the lower extremities functional status assessment using the Modified Lower Extremity Functional Scale obtained an average value of 60.93 , with a minimum value of 31 and maximum value of 84 from the total maximum value of 88 .

There was a significant correlation between severity of pain with functional status, with a value of $r=-.439$, and no significant correlation between radiographic grading of osteoarthritis and functional status (Table 2).

\section{Discussions}

The main problem due to damage to the joint cartilage in osteoarthritis is pain and disruption of related joint function. ${ }^{8}$ Activities such as climbing stairs, getting up from the chair, and running cause pain in people with knee osteoarthritis. ${ }^{9}$ Pain is a decisive reason why patients are seeking treatment. This study proved that all patients who were present in health care felt pain. This study found that $34.5 \%$ of the subjects reported mild pain, moderate pain $62.1 \%$, and $3.4 \%$ severe pain. 
Table 1 Study Subjects Characteristics

\begin{tabular}{llcc}
\hline \multicolumn{2}{c}{ Data Characteristics } & Total $\mathbf{n = 2 9}$ & $\mathbf{\%}$ \\
\hline Sex & Male & 1 & 3.4 \\
\multirow{3}{*}{ Diagnosis } & Female & 28 & 96.6 \\
& OA genu bilateral & 18 & 62.1 \\
& OA genu dekstra & 5 & 17.2 \\
Radiographic Grading of osteoarthritis & OA genu sinistra & 6 & 20.7 \\
(Kellgren-Lawrence Criteria) & I & 5 & 17.2 \\
& II & 23 & 79.3 \\
\multirow{4}{*}{ Severity of Pain } & III & 1 & 3.4 \\
& IV & 0 & 0 \\
& No pain (0) & 0 & 0.0 \\
& Mild pain (1-3) & 10 & 34.5 \\
& Moderate pain (4-6) & 18 & 62.1 \\
& Severe pain (7-10) & 1 & 3.4 \\
\hline
\end{tabular}

The variation of pain in osteoarthritis patients was explained through a study by Neogi et al. ${ }^{10}$, that one can adapt every day to overcome the pain and avoid activities that cause pain.

Based on the calculation performed in this study, there was a significant correlation between severity of pain with functional status, with a value of $r=-.439$ and $P<0.05$. This correlation showed the more severe pain experienced by the patients with knee osteoarthritis, the greater limitation of the lower extremity function. There was no significant correlation between the radiographic grading of osteoarthritis with lower extremity functional status in patients with osteoarthritis of the knee.

This study showed similar results with other studies even using the Modified Lower Extremity Functional as a measurement for functional status. Cubukcu et al..$^{5}$ conducted a study in Turkey using WOMAC to assess the grading of pain and functional status, reported the same results that pain on the knee affects the ability to perform daily activities in patients with osteoarthritis and there is no correlation between functional status and radiographic grading of osteoarthritis. A study performed by Ay and Evcik ${ }^{11}$ using VAS to assess the grading of pain and WOMAC to assess the functional status of the subject also had similar results. Another study also reported that pain affects the level of functional limitations in performing daily activities. ${ }^{12}$

The correlation between radiographic findings in osteoarthritis with functional status showed different results compared with the results of a research by Szebenyi et al. ${ }^{13}$ The radiological changes in the structure of the tibiofemoral and patellofemoral on the knee joints were associated with functional limitations in osteoartritis patients. This findings could be explained because in this study the radiological assessment of osteoarthritis only used the Kellgren-Lawrence criteria which did not assess more specifically, therefore the correlation found was different.

Furthermore, no correlation between the radiographic grading of osteoarthritis with functional status in this study could be found as $96.6 \%$ of the subjects of this study were

\section{Table 2 Correlation Test result}

\begin{tabular}{lcc}
\hline & \multicolumn{2}{c}{ Functional Status } \\
\cline { 2 - 3 } & Correlation Coefficient & Sig. \\
\hline Radiographic grading of osteoarthritis & -.226 & .239 \\
Severity of Pain & $-.439 *$ & $-.017^{*}$ \\
\hline
\end{tabular}

Note: *. Correlation is significant at the 0.05 level (2-tailed). 
women. Muraki et al. ${ }^{14}$ in Japan reported that the knee pain has a strong association with the radiological joint space narrowing, especially in men, and women more often feel pain even without radiological changes. Another study also stated that the pain severity is not correlated with the radiographic grading of osteoarthritis. ${ }^{5}$ Moreover, there is no correlation between severity of pain and radiographic grading of osteoarthritis.

A limitation of this study was the number of patients with osteoarthritis of the knee did not meet the minimum requirement number of samples so its significance couldnot be determined.

The conclusion of this study is the severity of pain correlates with the functional status of patients with osteoarthritis of the knee, while radiographic grading of osteoarthritis does not correlate with functional status.

\section{References}

1. Dorland WAN. Dorland's illustrated medical dictionary. 32th ed. Philadelphia, PA: Saunders; 2011.

2. Nainggolan O. Prevalensi dan determinan penyakit rematik di indonesia. Maj Kedokt Indon. 2009;59(12):588-94.

3. Dieppe PA, Lohmander LS. Pathogenesis and management of pain in osteoarthritis. Lancet. 2005;365(9463):965-73.

4. WHO. Chronic rheumatic conditions. Geneva. [cited 2014 February 11]. Available from: http://www.who.int/chp/ topics/rheumatic/en/.

5. Cubukcu D, Sarsan A, Alkan H. Relationships between pain, function and radiographic findings in osteoarthritis of the knee: a cross-sectional study. Arthritis. 2012;2012:984050.
6. Hoogeboom TJ, Bie RAd, Broeder AAd, Ende CHvd. The dutch lower extremity functional scale was highly reliable, valid and responsive in individuals with hip/ knee osteoarthritis. BMC Musculoskelet Disord. 2012;13(117):10.

7. Pua YH, Cowan SM, Wrigley TV, Bennell KL. The lower extremity functional scale could be an alternative to the western ontario and momaster universities osteoarthritis index physical function scale. J Clin Epidemiol. 2009;62(10):1103-11.

8. Enohumah KO, Imarengiaye CO. Pain in osteoarthritis. Afr J Biomed Res. 2008;11(2):119-28.

9. Felson DT. Osteoarthritis of the knee. N Engl J Med. 2006;354(8):841-8.

10. Neogi T, Nevitt MC, Yang M, Curtis JR, Torner J, Felson DT. Consistency of knee pain: correlates and association with function. Osteoarthr cartil. 2010;18(10):1250-5.

11. Ay S, Evcik D. Effectiveness of pain, disease severity and radiological grading on disability of daily living activities in knee osteoarthritis. Turk J Rheumatol. 2008;23(1):14-7.

12. Neogi $\mathrm{T}$. The epidemiology and impact of pain in osteoarthritis. Osteoarthr Cartil. 2013;21(9):1145-53.

13. Szebenyi B, Hollander AP, Dieppe P, Quilty B, Duddy J, Clarke S, et al. Associations between pain, function, and radiographic features in osteoarthritis of the knee. Arthritis Rheum. 2006;54(1):230-5.

14. Muraki S, Oka H, Akune T, Mabuchi A, En-yo Y, Yoshida M, et al. Prevalence of radiographic knee osteoarthritis and its association with knee pain in the elderly of japanese population-based cohorts: the ROAD study. Osteoarthr Cartil. 2009;17(9):1137-43. 